\title{
VALIDATION AND REGULARIZATION IN DIFFUSION MRI TRACTOGRAPHY
}

\author{
J. S. W. Campbell ${ }^{\S \dagger}$, P. Savadjiev ${ }^{\dagger}$, K. Siddiqi ${ }^{\dagger}$, and G. B. Pike $e^{\S}$ \\ $\S_{\text {Montreal Neurological Institute, } \mathrm{McGill} \text { University }}^{\dagger}{ }^{\dagger}$ Centre for Intelligent Machines, McGill University
}

\begin{abstract}
We present a physical phantom designed for fibre tractography validation and use it to evaluate tracking algorithms that employ (a) the classic diffusion tensor model of diffusion, (b) high angular resolution reconstruction of the diffusion orientation distribution function (ODF), and (c) a regularization algorithm capable of inferring complex subvoxel fibre configurations. This work addresses four issues in diffusion MRI tractography: validation of the tracking process using ground truth, evaluation of different approaches for diffusion ODF reconstruction, coping with imaging noise, and coping with confounding subvoxel fibre configurations.
\end{abstract}

\section{INTRODUCTION}

Fibre tractography concerns the reconstruction of possible white matter pathways which form the connections through which neuronal signals are conveyed in the brain. The delineation of these pathways is useful in determining whether specific areas of the brain are connected, the course of these connections, and how these change in disease. Prior to the advent of tractography using diffusion MRI, our knowledge of human neuronal connectivity was derived primarily from postmortem studies and tracer studies in nonhuman primates [1]. The first work on 3D fibre reconstruction using diffusion MRI used diffusion tensor (DT) data, so named because it is obtained by modeling the diffusion probability density function (pdf) as an anisotropic 3D Gaussian function, which can be described by a second order tensor [2]. The reconstruction of tracts was done by line propagation using the principal eigenvector of the diffusion tensor [3-5]. Such principal diffusion direction (PDD) techniques can be confounded when there is more than one fibre direction within a single imaging voxel. This partial volume averaging of fibre directions happens when tracts have high curvature, cross, branch, splay, or come to a bottleneck within an imaging voxel. The tensor case is a low angular resolution estimate of the diffusion pdf. High angular resolution approaches for estimating the diffusion orientation distribution function (ODF), which is obtained by integrating out the radial information in the pdf, include diffusion spectrum imaging (DSI) [6], q-ball imaging [7], and a composite hindered and restricted model of diffusion (CHARMED) [8].

Here, we present a phantom designed for fibre tractography validation and present comparisons between fibre tracking algorithms using diffusion tensor and high angular resolution diffusion ODF reconstruction. The phantom has known fibre structures that simulate common tract geometry in the human brain. Additionally, we present a regularization method that can be used for denoising ODFs with arbitrary angular resolution. The algorithm, which uses information from neighbouring voxels, can infer multiple maxima in the ODF obtained from diffusion tensor reconstruction, and can differentiate between cases of subvoxel partial volume averaging of directions that can confound even high angular resolution reconstructions: for example, crossing versus branching versus fibre pathways forming a bottleneck.

\section{A PHANTOM FOR EVALUATION OF DIFFUSION MRI TRACTOGRAPHY}

\subsection{Background}

Evaluation of diffusion MRI tractography methods in vivo is difficult because we do not have ground truth: details of connections vary from individual to individual, especially near the cortex, and the connectivity of some human fibre systems is still not well known. Tracer injection studies cannot be done in humans. Hence, having a phantom on which to test diffusion MRI acquisition and tractography is of significant value. Here, we present a physical biological phantom with known white matter connectivity for use in diffusion MRI validation studies. This allows us to evaluate the entire fibre tracking process: acquisition, diffusion ODF estimation, and fibre tracking algorithms, using a standard human brain MR scanner.

\subsection{Methods}

The phantom was constructed using freshly excised Sprague-Dawley rat spinal cords. Using white matter, instead of a synthetic material such as rayon [9], has the benefit of more closely simulating in vivo properties of restricted diffusion and exchange between compartments. The tissue was not fixed, as fixing tissue has been found to significantly reduce the magnitude of diffusion [10], even when rinsed with saline solution prior to scanning [11]. While this reduction in mean diffusivity may be acceptable for studies using small bore, high field systems, it significantly impairs the ability to precisely measure the diffusion pdf using a clinical MR system.

The phantom (Fig.1a) was designed to have fibre pathways with varying degrees of curvature that crossed in one region. The rats used were one year old, with spinal cords approximately $12.5 \mathrm{~cm}$ in length and $5 \mathrm{~mm}$ in diameter. Two rat spinal cords were embedded in $2 \%$ agar immediately after being surgically excised, and were scanned using a Siemens 1.5T Sonata MR scanner (Siemens Medical Systems, Erlangen, Germany) using a knee coil. A singleshot spin-echo echo planar diffusion weighted sequence was used. Eight coregistered datasets were acquired, consisting of 90 diffusion weighted images with isotropically spaced diffusion weighting directions each (four with $b=1300 \mathrm{~s} / \mathrm{mm}^{2}, 2.5 \mathrm{~mm}$ isotropic voxels, 40 slices, and four with $b=3000 \mathrm{~s} / \mathrm{mm}^{2} q=0.35 \mu \mathrm{m}^{-1}, 2.8 \mathrm{~mm}$ isotropic voxels, 30 slices; $\mathrm{TR}=8 \mathrm{~s}, \mathrm{TE}=110 \mathrm{~ms}$ ), as well as 10 images with $b=0 \mathrm{~s} / \mathrm{mm}^{2}$ and otherwise identical imaging parameters. These voxel sizes reflect standard values used in vivo: because the signal to noise ratio (SNR) per unit time is lower when higher $b$ values are used, spatial resolution is compromised. A $1 \mathrm{~mm}$ isotropic resolution $\mathrm{T}_{1}$-weighted gradient echo scan was also performed $(\mathrm{TR}=22 \mathrm{~ms}$, 
$\left.\mathrm{TE}=9.2 \mathrm{~ms}, \alpha=30^{\circ}\right)$. For all datasets, the diffusion tensor derived diffusion ODF was calculated. For the $b=3000 \mathrm{~s} / \mathrm{mm}^{2}$ datasets, the qball (QB) derived diffusion ODF was calculated.

The phantom can be used to perform extensive quantitative comparisons between fibre tracking algorithms [12]. Here, we present an example, qualitative tractography comparison between DT-PDD tracking and QB-PDD tracking. The $b=1300 \mathrm{~s} / \mathrm{mm}^{2}$ data were used for DT reconstruction. Streamline fibre tracking using FACT integration [3] was performed, starting in all voxels in the volume and retaining those pathways that passed through a region of interest (ROI) spanning a cross-section of the curved cord near its end. All maxima of the diffusion ODF that produced curves with radius of curvature $2.5 \mathrm{~mm}$ or greater were followed (directions that generated paths with higher curvature were assumed to be crossing fibres and were not followed). Tracking was constrained to voxels with fractional anisotropy (FA) of 0.05 or greater.

\subsection{Results and Discussion}

Fig.1 shows the DT and QB diffusion ODFs calculated using the $b=3000 \mathrm{~s} / \mathrm{mm}^{2}$ data. The ODFs have been stretched by squaring the sampled ODF values in order to accentuate anisotropy. There is support for a fibre running through the crossing region from bottom left to top right in the QB reconstruction, but streamline tracking using the principal eigenvector can easily be confounded by the presence of the fibre running from bottom right to top left. The diffusion ODF maps displayed imaging artifacts and noise characteristics typical of human in vivo diffusion experiments: eddy current induced field gradients gave rise to artifactual ODF maxima at the interface between the cords and the agar, and noise gave rise to variations in the ODF maxima from the ground truth direction.

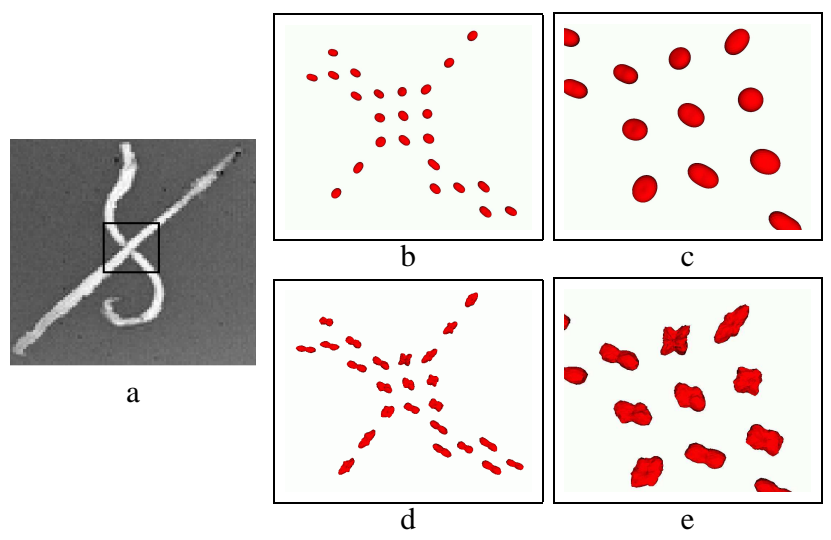

Fig. 1. Evidence of subvoxel partial volume averaging of fibre directions in the anisotropic diffusion phantom. (a) Crossing region outlined on T1-weighted scan of phantom. (b) DT reconstruction of the diffusion ODF. (c) Zoom of central region of DT data. (d) QB reconstruction from the same diffusion weighted images. (e) Zoom of central region of $\mathrm{QB}$ data.

Fig.2 shows the tracking results. The QB-PDD approach is able to pass through the crossing region, while the DT-PDD approach is not. We note that the tracking results may be improved by interpolating the data to a finer grid before tracking is performed, or by using a different integration scheme, such as RK4 integration. This example illustrates the difficulties posed by fibre crossing for a given set of tracking parameters.

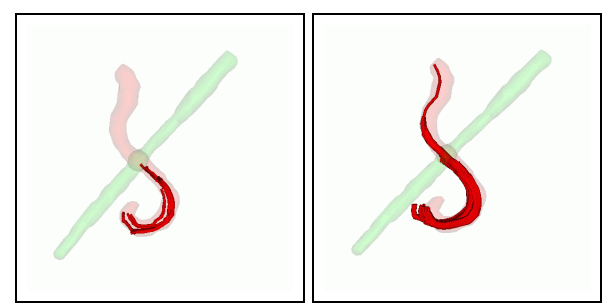

Fig. 2. Fibre tracking result in phantom using (LEFT) DT-PDD and (RIGHT) QB-PDD tracking. A transparent surface indicating the gold standard cord segmentation is shown for reference.

\section{CURVE INFERENCE FOR DIFFUSION MRI REGULARIZATION AND TRACKING}

\subsection{Background}

The potential advantage of high angular resolution reconstruction of the diffusion ODF in cases of crossing fibres was demonstrated in the above investigation in phantom. However, there are still cases in which the underlying fibre structure is ambiguous. These include distinguishing branching from crossing and distinguishing regions of crossing fibres from regions where fibres form a bottleneck. In these cases, considering information from a larger neighbourhood prior to tracking can be beneficial. Here, we describe an algorithm that infers which curves exist in each voxel, as opposed to which single fibre directions exist. Knowledge of which curves exist in each voxel can be used to distinguish those cases where even the high angular resolution diffusion ODF is ambiguous.

The curve inference algorithm has several other important applications. Inferring curves using information from neighbouring voxels can be used to regularize the data, and can be used to infer high angular resolution structure from lower angular resolution input data. The latter is of particular benefit when the available data is limited to diffusion tensor reconstruction, due to low $b$ values and/or sparse diffusion encoding directions, which may have been used to shorten scan duration. In many cases, a diffusion MRI scan may be acquired in combination with other MRI scans in a single session, and the scanning time required for high angular resolution diffusion scanning may be prohibitive. In these cases, the curve inference algorithm can be applied to the diffusion tensor field to infer multiple subvoxel fibre directions. Other approaches for inferring high angular resolution structure from diffusion tensor data include [13,14]. The curve inference algorithm can be applied to a diffusion ODF sampled at arbitrarily high angular resolution, and therefore can be used for denoising a high angular resolution diffusion ODF field as well.

\subsection{Methods}

The regularization algorithm [15] is an extension to 3D of the 2D curve inference algorithm of Parent and Zucker [16]. It models fi- 
bre tracts locally as segments of 3D helix curves, which have constant curvature and torsion. A tract is a concatenation of helix segments, and curvature and torsion can vary along the tract. The algorithm uses relaxation labeling [17] to maximize average local support for ODF directions. Relaxation labeling is a formal computational framework that allows a set of labels (here, the set of discretized ODF values) defined at separate nodes (here, voxels) to be assigned confidence values based on the support for these labels from neighbouring nodes (here, orientations that are part of helixsegments receive high support). For each voxel (i), for each of $N$ isotropic ODF sampling directions (u), the average local support $(s)$ is calculated by considering all pairs of voxels in a local neighbourhood of the voxel $i$. The average local support for direction $\mathbf{u}_{m}$ at voxel $i$ is given by

$$
s\left(\mathbf{u}_{m i}\right)=\sum_{j} \sum_{k} \sum_{n} \sum_{p} r_{i j k}\left(\mathbf{u}_{m} \quad \mathbf{u}_{n} \quad \mathbf{u}_{p}\right) \Psi_{j}\left(\mathbf{u}_{n}\right) \Psi_{k}\left(\mathbf{u}_{p}\right)
$$

where $j$ and $k$ are voxel indices that range over the local neighbourhood of voxel $i$ and $n$ and $p$ are direction indices that range over 1 to $\mathrm{N} . \Psi(\mathbf{u})$ is the ODF value in direction $\mathbf{u} . r_{i j k}$ is an index of cohelicity for the three orientations $\mathbf{u}_{m}, \mathbf{u}_{n}$, and $\mathbf{u}_{p}$ : it is equal to 1 if the three orientations are cohelical, and 0 if not. $\mathrm{N}$ can be arbitrarily high depending on the density of ODF samples desired in the output.

The regularization algorithm was run on both the phantom dataset described in section 2 and a human brain dataset. The human brain diffusion weighted images were acquired using a Siemens 3T Trio MR scanner. The acquisition used 99 diffusion encoding directions, $2 \mathrm{~mm}$ isotropic voxel size, 63 slices, and $b=3000 \mathrm{~s} / \mathrm{mm}^{2}$. Both the DT-ODF and QB-ODF were calculated using all of the data. A T1weighted anatomical scan was also acquired. For both datasets, streamline tractography was run as described in section 2.2.

\subsection{Results and discussion}

Fig.3a and Fig.3b show the DT derived diffusion ODF, using the $b=1300 \mathrm{~s} / \mathrm{mm}^{2}$ phantom data, and the output of the regularization algorithm using this ODF as input. The region shown is the same as that shown in Fig.1, but the spatial resolution is slightly higher. The regularization algorithm infers multiple ODF maxima in voxels dominated by one fibre direction in the DT ODF. Additionally, in voxels where the DT ODF maximum is intermediate to the two crossing fibre directions, the regularization algorithm is able to recover the two crossing directions. Fig. $3 \mathrm{c}$ shows the tracking result obtained using the same ROI as in Fig.2. The tracking is able to pass through the region of fibre crossing.

Fig.4 shows the DT ODF, the regularized DT ODF, and the QB ODF in an ROI containing fibres from the cortical spinal tract and corpus callosum in the human brain. This illustrates the case of fibres forming a bottleneck: both the QB and regularized DT ODFs exhibit multiple maxima in the region of partial volume averaging. Some blurring was observed in the regularized DT ODF map as compared to the QB ODF map.

Tractography results using a small ROI near the cortex are shown in Fig.5. The DT, QB and regularized DT reconstructions indicate U fibre structure near the cortex, but the QB and regularized DT tracking show more extensive cortico-cortical connections in this area. All three reconstruction approaches show part of the cortical spinal tract, but the DT approach does not fully reconstruct this pathway. The QB and regularized DT reconstructions also indicate a pathway from the ROI to the corpus callosum. It is probable that fibres from all of

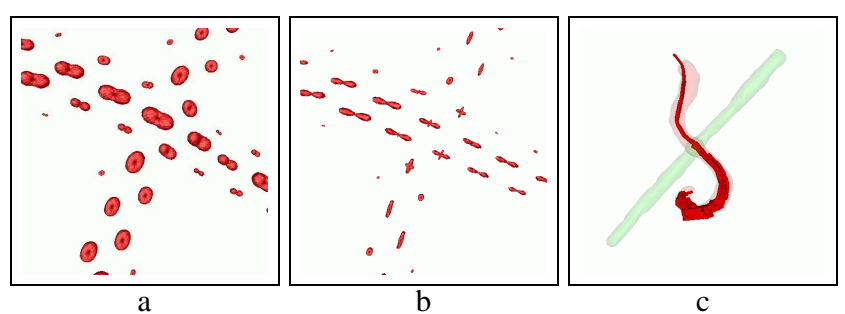

Fig. 3. (a) DT ODFs in the region of fibre crossing in the phantom. (b) Regularized result using the DT ODF as input. (c) Tracking result using regularized DT ODF.
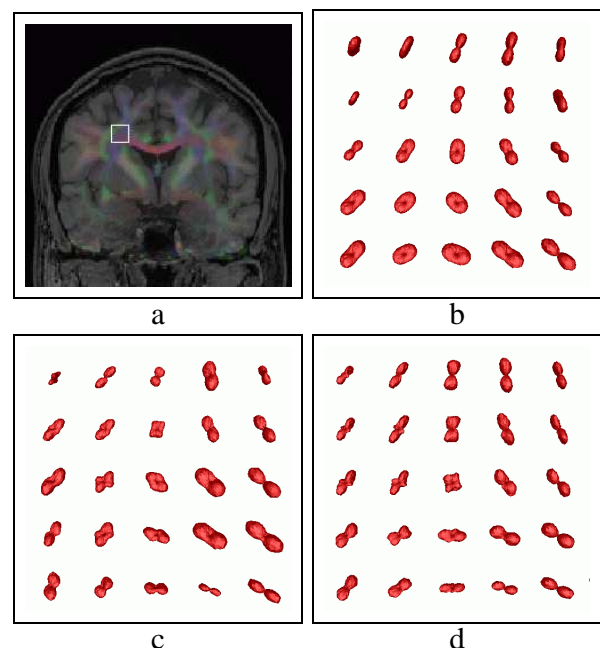

b

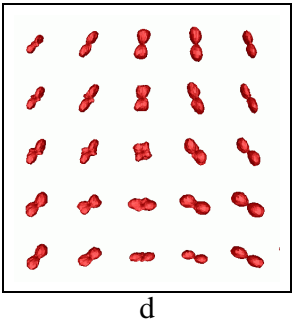

Fig. 4. ODFs obtained using (b) DT, (c) QB, and (d) regularized DT reconstructions in a region of partial volume averaging of cortical spinal tract and corpus callosum. The ROI is indicated by the white box in (a), which shows the principal diffusion direction RGB map overlaid on the T1 weighted anatomical image.

these pathways pass through the ROI. The high angular resolution of the QB reconstruction and the inferred high angular resolution of the regularized DT reconstruction allow the tracking to branch in regions where multiple fibre directions exist, giving more accurate information about the connectivity. In this example, as in the phantom, the regularized DT and QB approaches perform equally well, however, further investigation is required to determine in what cases regularized DT should be used in place of high angular resolution reconstruction of the diffusion ODF. Because of the loss of spatial resolution inherent in any smoothing algorithm, a high angular resolution acquisition may be indicated whenever time permits an acquisition with suitable SNR and resolution. The curve inference algorithm can still be used to distinguish between branching, crossing, and fibres forming a bottleneck, as discussed above. Additionally, we have not investigated the limits to the angular resolution achievable using curve inference. Here, we have matched the number of sampling directions used in the HARD acquisition to which we compare the results, however, it may be possible to use more sampling directions and achieve higher angular resolution. It could then be possible to use curve inference to refine existing HARD ODF estimates. 

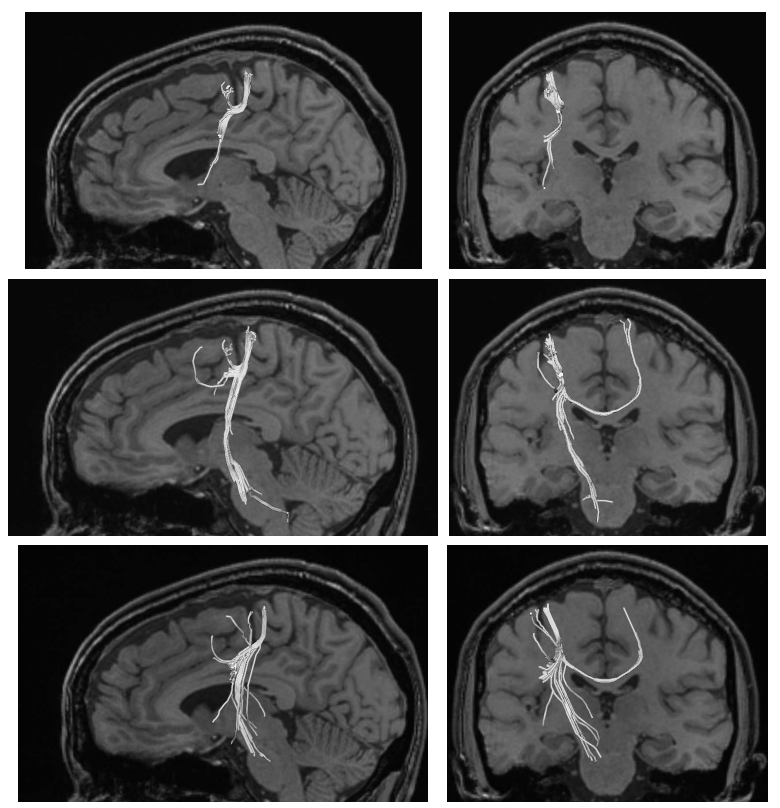

Fig. 5. Tractography results showing connections that pass through a small ROI near the cortex. The ODFs used to drive the tractography were TOP: DT, CENTRE: QB, and BOTTOM: regularized DT. A sagittal view is shown at left and a coronal view at right. The T1 weighted anatomical image is shown for reference.

\section{CONCLUSION}

We have presented a phantom useful for validation and comparison of fibre tracking algorithms. Using this phantom, we have illustrated the advantage of high angular resolution diffusion ODF reconstruction over the classical diffusion tensor method in the context of fibre tracking. We have presented a regularization algorithm that can be used for (a) inferring high angular resolution structure from diffusion tensor ODF fields, (b) denoising both diffusion tensor and high angular resolution ODF fields, and (c) extracting information about which curves are supported in each voxel. The performance of this algorithm has been demonstrated both in phantom and in vivo.

\section{ACKNOWLEDGMENTS}

The authors would like to thank Vladimir V. Rymar for performing the rat spinal cord dissection described in this paper.

\section{REFERENCES}

[1] M. Petrides and D.N. Pandya, Principles of frontal lobe function. Oxford University Press, 2002.

[2] P.J. Basser, J. Mattiello, and D. Le Bihan, "Estimation of the effective self-diffusion tensor from the NMR spin echo.," $J$. Magn. Reson., vol. 103, pp. 247-254, 1994.

[3] S. Mori, B.J. Crain, VP. Chacko, and P.C.M. van Zijl, "Three dimensional tracking of axonal projections in the brain by magnetic resonance imaging.", Ann. Neurol., vol. 45, pp. 265-269, 1999.
[4] P.J. Basser, S. Pajevic, C. Pierpaoli, J. Duda, and A. Aldroubi, "In vivo fiber tractography using DT-MRI data.," Magn. Reson. Med., vol. 44, pp. 625-632, 2000.

[5] T.E. Conturo, N.F. Lori, T.S. Cull, E. Akbudak, A. Snyder, L.S. Shimony, R.C. McKinstry, H. Burton, and M.E. Raichle, "Tracking neuronal fibre pathways in the living human brain.," PNAS, vol. 96, pp. 10422-10427, 1999.

[6] V.J. Wedeen, T.G. Reese, D.S. Tuch, M.R. Weigel, J.-G. Dou, R.M. Weiskoff, and Chessler D., "Mapping fibre orientation spectra in cerebral white matter with Fourier-transform diffusion MRI.," in Proc. $8^{\text {th }}$ ISMRM, 2000, p. 82.

[7] D. S. Tuch, T. G. Reese, M. R. Wiegell, and Van J Wedeen, "Diffusion MRI of complex neural architecture," Neuron, vol. 40, no. 5, pp. 885-95., Dec 42003.

[8] Y. Assaf and P. J. Basser, "Composite hindered and restricted model of diffusion (charmed) mr imaging of the human brain," NeuroImage, vol. 27, no. 1, pp. 48-58., 2005.

[9] P. Scifo, F. Dell' Acqua, G. Rizzo, M. Gilardi, and F. Fazio, "A dedicated phantom for diffusion imaging studies.," in Proc. $12^{\text {th }}$ ISMRM, 2004, p. 1203.

[10] S. W. Sun, J. J. Neil, and S. K. Song, "Relative indices of water diffusion anisotropy are equivalent in live and formalinfixed mouse brains," Magn. Reson. Med., vol. 50, no. 4, pp. 743-8., 2003.

[11] E. T. Ahrens, D. H. Laidlaw, C. Readhead, C. F. Brosnan, S. E. Fraser, and R. E. Jacobs, "MR microscopy of transgenic mice that spontaneously acquire experimental allergic encephalomyelitis," Magn. Reson. Med., vol. 40, no. 1, pp. 119-32., 1998.

[12] J.S.W. Campbell, K. Siddiqi, V.V. Rymar, A.F. Sadikot, and G.B. Pike, "Flow-based fiber tracking with diffusion tensor and q-ball data: Validation and comparison to principal diffusion direction techniques," NeuroImage, vol. 27, no. 4, pp. 725736, 2005.

[13] Y. Cointepas, C. Poupon, D. Le Bihan, and J.-F. Mangin, "A spin glass framework to untangle fiber crossing in MR diffusion based tracking," in MICCAI 2002, LNCS 2488, 2002, pp. 475-482.

[14] A. Ramírez-Manzanares and M. Rivera, "Brain nerve bundles estimation by restoring and filtering intra-voxel information in diffusion tensor MRI," in Proc. 2nd IEEE VGLSM, 2003.

[15] P. Savadjiev, J.S.W. Campbell, G.B. Pike, and K. Siddiqi, "3D curve inference for diffusion MRI regularization," in MICCAI 2005, Part I, LNCS 3749, 2005, pp. 123-130.

[16] P. Parent and S.W. Zucker, "Trace inference, curvature consistency, and curve detection," IEEE PAMI, 1989.

[17] R.A. Hummel and S.W. Zucker, "On the foundations of relaxation labeling processes," IEEE PAMI, 1983. 